\title{
A Study of the Correlation between VEP and Clinical Severity in Children with Autism Spectrum Disorder
}

\author{
Winai Sayorwan, ${ }^{1}$ Nutthida Phianchana, ${ }^{2}$ \\ Kannika Permpoonputtana, ${ }^{3}$ and Vorasith Siripornpanich ${ }^{2}{ }^{2}$ \\ ${ }^{1}$ Kanchanabhishek Institute of Medical and Public Health Technology, Nonthaburi 11150, Thailand \\ ${ }^{2}$ Research Center for Neuroscience, Institute of Molecular Biosciences, Mahidol University, Nakhon Pathom 73170, Thailand \\ ${ }^{3}$ Department of Occupational Therapy, Faculty of Physical Therapy, Mahidol University, Nakhon Pathom 73170, Thailand \\ Correspondence should be addressed to Vorasith Siripornpanich; drvorasith@gmail.com
}

Received 25 September 2017; Revised 1 December 2017; Accepted 14 December 2017; Published 14 January 2018

Academic Editor: Klaus-Peter Ossenkopp

Copyright (C) 2018 Winai Sayorwan et al. This is an open access article distributed under the Creative Commons Attribution License, which permits unrestricted use, distribution, and reproduction in any medium, provided the original work is properly cited.

\begin{abstract}
Visual evoked potential (VEP) is a technique used to assess the brain's electrical response to visual stimuli. The aims of this study were to examine neural transmission within the visual pathway through VEP testing in preschool children with autism spectrum disorder (ASD) and compare it to age-matched controls, as well as search for a correlation between the VEP parameters and the symptoms of ASD. Participants were composed of ASD children ( 9 males) and typically developing children ( 8 males and 4 females), aged between 3 and 5 years. Checkerboards were chosen as the pattern-reversal VEP. The clinical severity of ASD was assessed using the Autism Treatment Evaluation Checklist (ATEC) and the Vineland Adaptive Behavior Scales 2nd edition (VABS-II). Our findings demonstrated that children with ASD had significantly longer N145 latency compared to the controls. A longer N145 latency correlated with a higher score of ATEC within the sensory/cognitive awareness subdomain. In addition, a slower N145 response was also associated with a lower VABS-II score within the socialization domain. The correlation between longer VEP latency and abnormal behaviors in children with ASD suggests a delayed neural communication within other neural circuits, apart from the visual pathway. These lines of evidence support the possibility of using VEP, along with clinical parameters, for the assessment of ASD severity.
\end{abstract}

\section{Introduction}

Autism is a common neurodevelopmental disorder characterized by impaired communication, deficits in social skills, and abnormal behaviors. The current version of the Diagnostic and Statistical Manual of Mental Disorders (DSM) by the American Psychiatric Association (APA), the DSM5 , collapses various diseases within the group of pervasive developmental disorders (PDD), including autism disorder, into a single disorder, the autism spectrum disorder (ASD) [1]. The diagnosis of ASD is strongly dependent on clinical assessment via a medical interview with the parents or a primary caregiver, as well as a direct observation of the child's behaviors within the exam room. There are, however, no available scientific tools for the evaluation of the clinical symptoms in children with ASD, which may lead to an underor an overestimation of the disease severity.
The well-accepted pathogenesis of autism is the abnormality in neural communication [2]. Impaired long-distance brain connectivity is also commonly observed in a number of autism researches [3-5]. An evaluation of brain connectivity is usually performed by a magnetic resonance imaging (MRI) based technique called diffusion tensor imaging (DTI). However, this neuroimaging technique requires an experienced neuroradiologist to perform and to interpret the results, demanding much time for data analysis, and is relatively quite expensive. In addition, DTI also requires subjects to limit their movements. It is, therefore, a rather difficult technique to perform on children with autism. Moreover, evidence from several researches shows abnormalities not only in structural but also in functional connectivity in children with autism [6].

Normal brain function not only requires an appropriate level of synaptic contacts, but also an appropriate speed 
of neural transduction. Increased electrical conduction is strongly dependent on the presence of myelin around the nerve fibers. Myelin is a fatty substance derived from the plasma membrane of glial cells and is crucial for rapid neural communication. Myelination, the production of myelin sheath around the axons, is an important brain maturation process which first occurs within the motor and sensory pathways, including the visual pathway, since the early developmental period.

Visual evoked potential (VEP) is an electrophysiological method used in measuring the brain electrical signals recorded over the occipital lobe in response to basic visual stimuli. Generally, VEP is used for the determination of optic nerve lesions, as well as in the evaluation of the visual functions. In clinical practice, VEP is commonly used to reveal the demyelination of optic nerves that is commonly seen in patients with optic neuritis and multiple sclerosis [7]. However, several studies have also revealed VEP abnormalities in various neurological and psychiatric disorders such as Alzheimer's disease $[8,9]$, migraine headache, and schizophrenia [10-12]. Interestingly, the abnormalities of VEP can also be found in children with developmental delays. A previous study has shown prolonged VEP latencies in slow learners [13]. In children with attention deficit hyperactivity disorder (ADHD), the VEP waveforms are slightly more prolonged than in healthy children [14]. Moreover, alterations in the VEP waveforms were also found in patients with dyslexia [15] and fragile $\mathrm{X}$ syndrome [16]. The prolongation of the VEP waveforms usually reflects delayed nerve conduction, which is commonly observed in children with developmental delays.

Children with autism usually show some abnormalities in the visual system such as poor eye control and hypersensitivity to light. These abnormal findings may result either from atypical sensory processing or from defects in neural networks of social cognition. A recent study using a steadystate VEP showed hyperresponsivity to visual stimuli in patients with high-functioning autism [17]. However, there is a current lack of studies that use VEP testing in children with ASD as a brain maturation index. To determine its correlation with potential clinical symptoms of ASD, we therefore planned to investigate neural transmission within the visual system using VEP as an index of the myelination process of the visual pathway and its correlation to the clinical severity of autism. The research hypothesis is that children with ASD show a delayed latency of the VEP waveforms compared to normal children. Moreover, the prolongation of VEP in children with ASD should correlate with some aspects of developmental delay in autism, which can provide a benefit to the VEP in assessing the clinical symptoms of autism.

\section{Methods}

2.1. Participants. The participants in this study consisted of children with ASD and typically developed children (controls). The total number of subjects, aged between 3 and 5 years, was 21: 9 children with ASD (all males) and 12 agematched controls ( 8 males: 4 females). The diagnosis of ASD in the current work was made during the recruitment period of the participants by a developmental behavioral pediatrician or a child and adolescent psychiatrist in accordance with the DSM-IV-TR, the latest version of DSM at the recruitment period [18].

Children with ASD in this study were initially screened for comorbid conditions. The exclusion criteria for this work included (1) genetic syndromes associated with autistic features such as tuberous sclerosis, (2) autism with neurodegenerative conditions such as Rett's syndrome and childhood disintegrative disorder, (3) patients with other psychiatric disorders, (4) cases with active epilepsy, (5) impaired gross motor function, and (6) the taking of benzodiazepines. Any participant who passed the initial screening process was recruited to receive a complete systemic and neurological examination by a pediatric neurologist (VS). Subjects who had abnormal eye signs such as the presence of nystagmus, significant myopia, and abnormal retinal examination, as well as the presence of focal neurological deficits, were excluded from this study. In addition, handedness was assessed using the Edinburgh Handedness Inventory for the determination of the participants' hand dominance in both groups [19]. Only right-handed children were selected for this study.

All experiments in this study were conducted in accordance with the Declaration of Helsinki and approved by the ethical standards of the Mahidol University Central Institutional Review Board (MU Central-IRB). The research project was given ethical permission number MU-IRB 2013/163.1712. On the date of experimentation, the whole protocol was clearly explained to the parents of all the participants in both groups. They had to sign written consent forms before the experiment could commence. The parents were clearly informed that the experiment could be terminated anytime if their children felt uncomfortable or could not maintain their cooperation.

2.2. Evaluation of the Clinical Severity of ASD. In Thailand, the Pervasive Developmental Disorder Screening Questionnaire (PDDSQ) was developed by the Yuwaprasart Waithayopathum Child Psychiatric Hospital of Thailand to be used as a screening tool for children with ASD [20]. It was adapted from several assessment tools for ASD, for example, from the Checklist for Autism in Toddlers (CHAT), the Childhood Autism Rating Scale (CARS), the Autism Screening Questionnaire (ASQ), and the Social Responsiveness Scale (SRS). PDDSQ was established as a checklist for parents or primary caregivers to evaluate the behaviors of their children aged between 1 and 18 years. In this study, all children were screened for history of PDD using this questionnaire.

The clinical severity of autism was evaluated using the Autism Treatment Evaluation Checklist (ATEC). The ATEC was created by Rimland and Edelson in 1999 and was translated into a Thai version with permission in 2004 [21, 22]. The ATEC checklist has 4 subtests: speech/language communication, sociability, sensory/cognitive awareness, and health/physical/behavior domains. The severity scale of ATEC was divided into 3 levels of severity: mild, moderate, and severe. ASD children with severe clinical symptoms were excluded from the current work due to the high chance 
of poor cooperation. Importantly, regarding the purpose of assessing the clinical severity of autism, ATEC was completed only in the ASD group but not in the control group.

In regard to social development, which is one of the key deficits in children with ASD, the Vineland Adaptive Behavior Scales 2nd edition (VABS-II) was used to assess the adaptive behavior skills of the children [23]. VABS-II is a semistructured interview with a parent, primary caregiver, or teacher. There are 4 domains in VABS-II: communication, daily living skills, socialization, and motor skills. However, only the socialization domain was selected to evaluate the level of social development of all participants. The socialization domain contains three subdomains: interpersonal relationships, play and leisure time, and coping skills. On the contrary to ATEC, children with ASD as well as typically developing children were evaluated by VABS-II for social adaptive behaviors.

2.3. Visual Evoked Potential (VEP). VEP is the electrophysiological method of evaluating the integrity of the visual pathway. However, researches using VEP in patients with brain developmental disorders have also been reported. There are two main types of VEP that are commonly used in clinical studies: flash VEP and pattern-reversal VEP. For the current study, we selected the pattern-reversal VEP because it is more suitable for VEP recording in young children, especially in cases with high degree motor activities and poor inhibitory control. The black and white checkerboard was evenly presented in horizontal grates at a viewing distance of $60 \mathrm{~cm}$ from the computer monitor. The checkerboard was presented using the STIM 2.4 software (Neurosoft Inc.). The checkerboard pattern consisted of $8 \times 8$ checks, including each individual check being subtended at $2.75^{\circ}$ visual angle $(100 \%$ contrast). According to the visual pattern-reversal program, the reversal interval value was set at 500 milliseconds, resulting in a reversal every 0.5 seconds. All participants were prompted to focus on a red-cross fixation point at the center of the display screen during the 3-minute contrast-reversing checkerboard presentation.

VEP Acquisition. For VEP recording, a full setup of the electroencephalography (EEG) and evoked potential (EP) was performed. The EEG recording system used in this experiment was the Neuroscan version 4.3 (Neurosoft Inc.). The electrodes were attached onto the Electro-Cap according to the international 10-20 system, with 4 additional electrodes for the electrooculography (EOG) recording [24]. Both mastoid regions were used as reference sites. QuikCell, a cellulose based transmission, was inserted into all 32 channels attached on the Electro-Cap, followed by the use of a blunt needle tip to drop in liquid electrolytes for cell hydration. After the QuikCells were hydrated, they acted as a conducting bridge. The impedance was kept below $10 \mathrm{k} \Omega$. The prerecording band-pass filter was set from 0.1 to $60 \mathrm{~Hz}$. A notch was opened at $50 \mathrm{~Hz}$. The EEG and the EOG were recorded at a sampling rate of $1,024 \mathrm{~Hz}$.

Analysis of the VEP Waveforms. In regard to the analysis of the VEP waveforms, the main electrodes used for the analysis consisted of the $\mathrm{Oz}, \mathrm{O} 1$, and $\mathrm{O} 2$ electrodes, representing the midline, left, and right occipital regions, respectively. Artifact rejection was set to reject the epoch with the channel amplitude exceeding \pm 80 microvolts. The EEG segments time-locked to the onset of visual stimuli were selected from 100 milliseconds (ms) before and $300 \mathrm{~ms}$ after the stimulus onset. At least 80 epochs of artifact-free EEG data from each subject were allowed for further steps of VEP analysis. The period of EEG segment prior to the stimulus onset was used as baseline and the postrecording band-pass filter was assigned from 0.3 to $30 \mathrm{~Hz}$. VEP data was analyzed in the time domain. The amplitude and latency of the VEP waveforms were used as a basis of comparison between groups. Amplitude (microvolt) is defined as the difference between the height of the current peak and the highest point of the previous peak, while latency (millisecond) is defined as the duration from the visual stimulus to the peak of each VEP waveform. In general, the VEP waveforms generated in the pattern-reversal VEP consist of N75, P100, and N145 waves.

2.4. Statistical Analysis. All data generated from this study was statistically proven for normal distribution using the Kolmogorov-Smirnov test. The comparisons of the VEP waveforms were analyzed using independent-samples $t$-test between groups (ASD versus control). In addition, Pearson's correlation was used to assess the correlation within the ASD group between the VEP parameters and the ATEC scores, as well as the correlation between the VEP parameters and the behavioral score of VABS-II in social performance for all participants.

\section{Results}

The age distribution in both groups was not statistically different by means of the Kolmogorov-Smirnov test. There was also no significant difference in terms of the handedness and the socioeconomic profiles. As expected, the scores of PDDSQ and VABS-II, especially in the socialization domain, were significantly different $(p<0.05)$ between the two groups. Individuals with ASD had higher PDDSQ scores than controls, which is consistent with the prediction along the lines of the autistic trend. In addition, the ASD group showed lower scores than controls on the VABS-II subtest in the socialization domain, which explains their below-average social behaviors compared to children in their age group. The clinical characteristics of the participants including age, gender, handedness, and behavior screening scores are displayed in Table 1.

For the VEP analysis, the amplitude and the latency of the VEP waveforms were used as parameters in comparing the outcomes between the groups. At the site of the Oz electrode, our results showed that the latency of the N145 wave was significantly prolonged in the autism group, compared to the control group ( $\mathrm{df}=19, p=0.014$ ). In addition, the latency of the P100 wave was slightly prolonged in the autism group but did not reach the significant level $(\mathrm{df}=19, p=$ 0.08). Regarding the amplitude, the amplitude of the N75$\mathrm{P} 100$ wave in the autism group slightly reduced $(\mathrm{df}=19$, $p=0.51$ ), compared to the controls. All the VEP parameters 
TABLE 1: The clinical characteristics of children in both the ASD and the control groups. Data is shown as mean and standard deviation (SD).

\begin{tabular}{lcc}
\hline Clinical parameters & $\begin{array}{c}\text { ASD group } \\
(n=9)\end{array}$ & $\begin{array}{c}\text { Control group } \\
(n=12)\end{array}$ \\
\hline Age (months) & $47.44(9.98)$ & $45.5(8.44)$ \\
Gender ratio & $9: 0$ & $8: 4$ \\
(male : female) & $70.72(37.46)$ & $84.07(20.12)$ \\
Handedness (\%) & $27.33(4.64)^{*}$ & $42.92(4.12)$ \\
VABS socialization (total) & $20(6.98)^{*}$ & $4.42(3.58)$ \\
PDDSQ (total) & $66.44(15.72)$ & - \\
ATEC (total) & $(50-59)$ & \\
(percentile) & & \\
\hline
\end{tabular}
${ }^{*} p<0.05$.

TABLE 2: The amplitude and latency of the VEP waveforms over the Oz electrode between the two groups.

\begin{tabular}{lcc}
\hline $\begin{array}{l}\text { VEP waveform } \\
\text { (Oz electrode) }\end{array}$ & $\begin{array}{c}\text { Autism group } \\
(n=9)\end{array}$ & $\begin{array}{c}\text { Control group } \\
(n=12)\end{array}$ \\
\hline N75 latency & $79.11(8.77)$ & $79.91(12.43)$ \\
P100 latency & $131.89(25.19)$ & $115.75(14.85)$ \\
N145 latency & $192.44(32.7)^{*}$ & $161.5(19.87)$ \\
N75-P100 amplitude & $10.97(5.59)$ & $13.24(8.73)$ \\
P100-N145 amplitude & $12.65(6.92)$ & $13.44(6.83)$ \\
\hline
\end{tabular}

${ }^{*} p<0.05$.

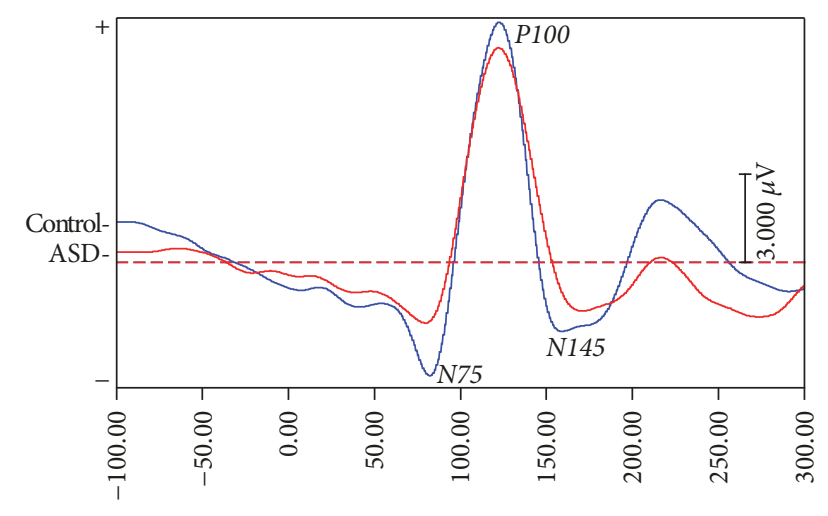

(ms)

$$
\begin{aligned}
& \text { Oz electrode } \\
& - \text { ASD } \\
& \text { Control }
\end{aligned}
$$

Figure 1: The VEP waveforms over the Oz electrode, a comparison between the ASD and the control groups.

are displayed in Table 2 and the VEP waveforms generated at the site of the $\mathrm{Oz}$ electrode are shown in Figure 1.

Apart from the Oz electrode, the latency of the N145 wave recorded over the $\mathrm{O} 2$ electrode in the autism group was longer than in the control group $(\mathrm{df}=19, p=0.02)$. Other parameters did not reveal a significant difference between the two groups. The VEP waveforms generated at the $\mathrm{O} 1$ and $\mathrm{O} 2$ electrodes are shown in Table 3.

The relationships between the VEP parameters and the ATEC scores were evaluated using Pearson's correlation only

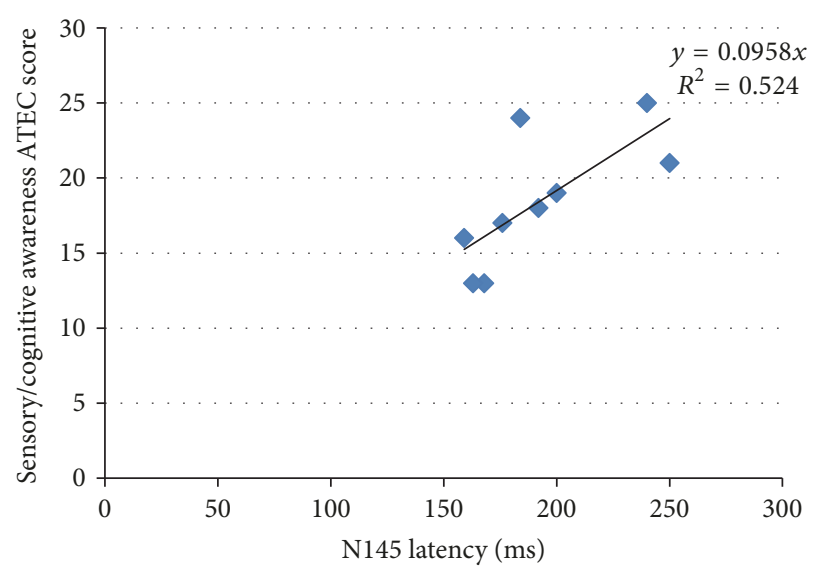

FIGURE 2: Scatter plot displaying the correlation between N145 latency over $\mathrm{Oz}$ electrode and the ATEC scores for subtests in sensory/cognitive awareness.

in the ASD group (Table 4). There was a positive significant correlation between N145 latency and ATEC score. Longer N145 latency was associated with a higher score within the sensory/cognitive awareness subdomain as measured using ATEC assessment $(r=0.724, p=0.03)$ as shown in Figure 2. These findings indicate that more severe autistic symptoms are correlated with a delayed VEP response in the N145 wave over the $\mathrm{Oz}$ electrode. However, no such correlation was observed between other VEP waveforms (N75 and P100 waves) and other subdomains of ATEC.

Across the entire body of participants, the latencies of the $\mathrm{P} 100$ and the N145 waves over the Oz electrode were found 
TABLE 3: A comparison between the VEP waveforms generated in each hemisphere, at the O1 electrode site for left occipital and the O2 electrode site for right occipital areas.

\begin{tabular}{lccr}
\hline VEP waveforms & Electrode & Autism group & Control group \\
\hline \multirow{2}{*}{ N75 latency } & O1 & $82.56(13.44)$ & $81(21.24)$ \\
& O2 & $81(10.11)$ & $80.72(17.53)$ \\
P100 latency & O1 & $127.44(12.72)$ & $112.75(21.11)$ \\
& O2 & $138.78(35.16)$ & $120.83(15.56)$ \\
N145 latency & O1 & $179.44(26.13)$ & $163.83(16.28)$ \\
N75-P100 amplitude & O2 & $192.63(35.77)^{*}$ & $161.58(19.76)$ \\
& O1 & $5.59(1.67)$ & $5.88(3.27)$ \\
P100-N145 amplitude & O2 & $9.75(5.96)$ & $10.37(5.89)$ \\
& O1 & $7.28(3.11)$ & $7.36(3.58)$ \\
\hline
\end{tabular}

${ }^{*} p<0.05$.

TABLE 4: A correlation analysis between the VEP parameters over the Oz electrode and the ATEC scores.

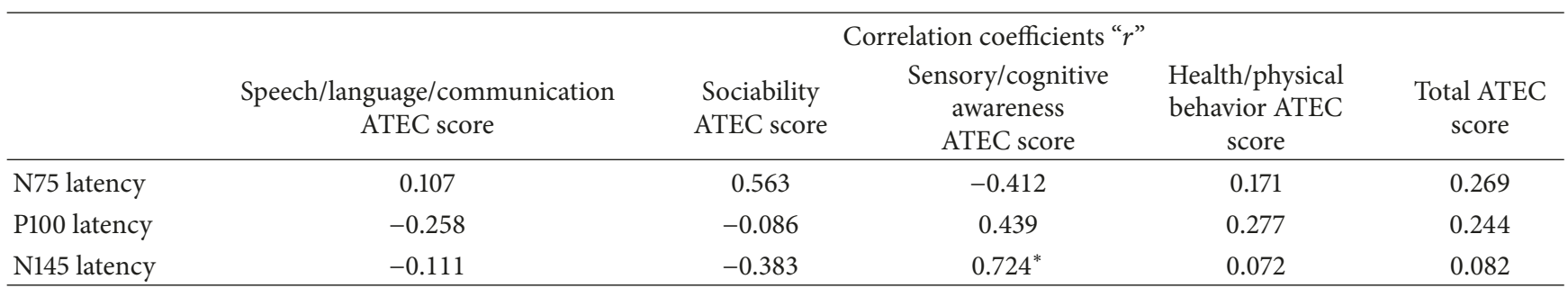

Note. ${ }^{*} p<0.05$.

to correlate with the standard scores measured using VABSII. In this study, the standard scores of VABS-II were focused on the socialization domain. On the interpretive scores, the lower socialization domain standard scores of VABS-II were described as more delayed in social interaction performance as well as more severe symptoms of social deficits. The higher adaptive behavior scores in the socialization domain reflected the better developmentally appropriate social functioning of children. There was a negative significant correlation between the P100 latency and the VABS-II scores only in the subdomain of socialization. Specifically, the longer P100 latency correlated with a lower score within the interpersonal relationships subdomain $(r=-0.436, p=0.048)$, but not in other subdomains. Interestingly, the correlation between N145 latency and VABS-II scores was negatively significant for the socialization domain $(r=-0.565, p=0.008)$ and all its subdomains, consisting of interpersonal relationships $(r=-0.539, p=0.012)$, play and leisure time $(r=-0.565$, $p=0.008)$, and coping skills $(r=-0.476, p=0.03)$ as shown in Table 5 and Figure 3.

\section{Discussion}

In this study, we investigate the neural transmission within the visual system, measured using pattern-reversal VEP in children with ASD, compared to age-matched controls. VEP is a method used in studying the function of the visual pathway and can be performed in poorly cooperative participants like children with ASD. The amplitude and the latency of VEP waveforms are used as the main parameters

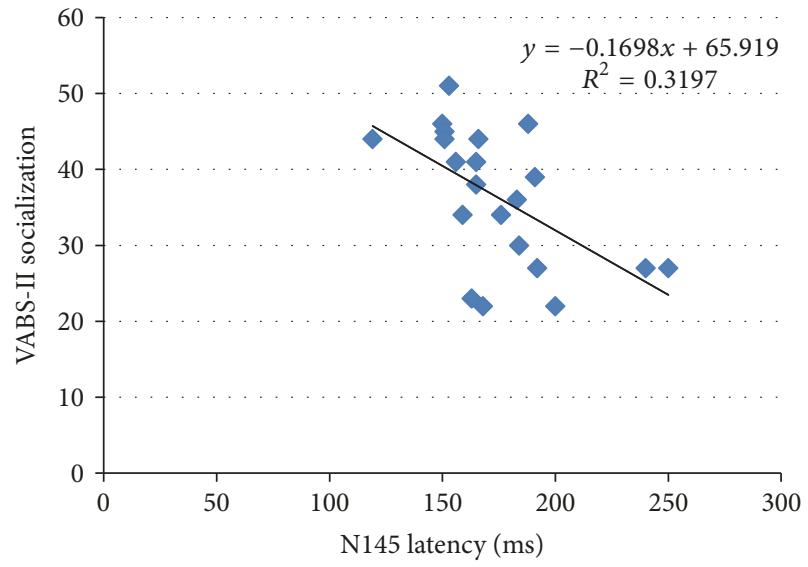

Figure 3: Scatter plot displaying the correlation between N145 latency over $\mathrm{Oz}$ electrode and VABS-II standard scores in the socialization domain.

for comparison. The latency of the VEP waveform indicates the velocity of the neural transmission in the optic nerve, a part of the visual pathway. Previous research showed that prolonged latency of the VEP waveforms is commonly found in patients with demyelination of the optic nerve, including patients with optic neuritis and multiple sclerosis $[25,26]$. Thus, myelination is the most important factor for determining the latency of the VEP waveforms. However, the physiological factors including age, gender, visual acuity, and medications as well as the cognitive factors have been 
TABLE 5: A correlation analysis between the VEP parameters over the Oz electrode and the VABS-II standard scores in the socialization domain and its subdomains.

Correlation coefficients " $r$ "

Interpersonal relationships subdomain Play and leisure time subdomain Coping skills subdomain Socialization domain

\begin{tabular}{lcccc}
\hline N75 latency & -0.001 & 0.015 & 0.022 & 0.007 \\
P100 latency & $-0.436^{*}$ & -0.319 & -0.321 & -0.392 \\
N145 latency & $-0.539^{*}$ & $-0.565^{* *}$ & $-0.476^{*}$ & $-0.565^{* *}$ \\
\hline
\end{tabular}

Note. ${ }^{*} p<0.05 ;{ }^{* *} p<0.01$.

reported to affect the VEP latency [27, 28]. In this study, we examined the neural communication within the visual pathway to evaluate the level of myelination of the visual circuit, and we tried to determine the possible correlations between the VEP parameters and the ASD symptoms for the possibility of using VEP as a method in the clinical evaluation of ASD severity.

Myelination is a process of brain maturation, which occurs early in life and continues to develop until early adulthood $[29,30]$. The pattern of myelination in the brain occurs in a region-specific manner [30]. In general, myelination begins in the sensory pathways, which include the visual system, followed by the motor pathways. The presence of myelin around the neuronal axons promotes the speed of neural transmission. In the visual pathway, myelination usually develops since early life, similar to the neural circuits in other sensory domains. Thus, if the visual function is normal, VEP can be used for the evaluation of the degree of myelination in early childhood, which corresponds with the maturation of neural communication.

A previous study using VEP in adults with ASD revealed an alteration in the VEP response, compared to controls. More specifically, a visual event-related potential (ERP) study in adults with Asperger's syndrome showed an alteration of early and late visual processing shown by the reduced amplitude of ERP waves [31]. Similarly, our study demonstrated the occurrence of different VEP signals in children with ASD, compared to typically developing (TD) children. The latency of N145 wave in children with autism was significantly longer over the $\mathrm{Oz}$ and $\mathrm{O} 2$ electrodes compared to age-matched controls. The prolonged N145 latency indicates delayed neural processing in the visual pathway. Some children with ASD even revealed some abnormality in the eyes and optic pathways that might affect their VEP results. Our study tried to reduce this confounding factor by excluding any participants who had abnormal eye signs. This elimination approach can help us confirm that delayed N145 latency is due to the slower neural communication in the visual circuit, but not because of any ocular or optic pathway lesions, which can be found in children with ASD.

Interestingly, the prolongation of VEP latency found in this current work correlates with higher ATEC scores within the sensory and cognitive awareness domains. The ATEC score is the measurement of the clinical severity of autism; therefore, this finding could be considered in linking clinical symptoms of autism with the electrophysiological parameters. It appears in this study that the N145 latency was slower in children with autism than in the TD children, which supports the delay of neural communication along the visual pathway. The correlation between delayed neural processing in visual pathway as shown by prolonged N145 latency and higher level of autism severity through ATEC scoring may indicate a similar brain maturation pattern between the visual pathway and the neural circuits within other sensory domains and cognition. This evidence supports our hypotheses in relation to the delayed neural transmission of the visual pathway in ASD children and the possibility of using VEP in evaluating the clinical severity of autism.

Moreover, the VEP response to checkerboards in children with ASD also showed that slower P100 and N145 latency over the $\mathrm{Oz}$ electrode correlated with lower VABS-II scores within the socialization domain. This finding indicates the correlation between longer VEP latencies and more delays in social interaction performance as shown by lower VABSII scores. Although there are a few studies that show correlations between the VEP electrophysiology recordings and the social behavior measurement in autism, the studies using ERP response to human face as the index of the N170 wave have been found to be consistent with social performance in children with autism. Among twins with and without autism, the speed of face processing as indicated by the latency of P1 and N170 waves was found to correlate with social skills. Faster P1 latency is associated with better social participation and greater response of the N170 wave to upright face images, which is related to fewer social difficulties [32].

Some studies have reported the alteration of amplitude of the VEP waveforms in patients with ASD, compared to children with normal developmental milestones. For example, a study using the novel transient visual evoked potential revealed that children with ASD showed smaller VEP amplitude than controls, while there was no group difference between the VEP latencies using this technique [33]. However, our findings did not demonstrate any significant alterations in the VEP amplitude between children with ASD and TD, with the VEP latency being the only parameter with a significant difference. The reason may be explained by the observation that the visual function in both groups of participants was normal, so the amplitudes of the VEP waveforms representing the neural activities within the visual pathway were not significantly different between the groups. The main difference is the speed of neural transmission as revealed by the longer VEP latency in children with ASD. Moreover, the correlations between the VEP amplitude and the severity of the ATEC scores, including social adaptive behavior, were also not observed in this study. 
There are some limitations to this study. First, the sample size in this study is rather small. Second, the gender distribution is different between the two groups. There are only males in the ASD group, whereas children in the control group consisted of both genders. Previous studies have demonstrated different VEP waveforms between males and females for both children and adults [27, 34, 35]; however, the unequal gender distribution observed in our work may affect the VEP results. Third, the cognitive factors, especially for attention, have been found to affect the latency of EP as well as ERP waveforms [28]. Thus, a formal assessment with neuropsychological tests should be performed in a further study. Finally, even with careful data analysis, the movement and muscle artifacts may have still persisted in the cases of poorly cooperative young children, particularly with the ASD participants.

\section{Conclusion}

Using VEP as the index of neural processing within the visual system of children with ASD could become a possible tool for the clinical assessment of the disease severity. The prolongation of N145 latency indicates delayed neural communication within the visual pathway. The correlation between VEP latency and the ASD clinical symptoms assessed using the ATEC and the VABS-II scores in the socialization domain may indicate a close association between myelination within the visual circuits and the symptoms of autism. More research evidence is, however, required to confirm the utility of VEP in clinical settings. Further research would also shed light on the technique of electrophysiological evaluation of the ASD symptoms, other than the routine clinical assessments.

\section{Conflicts of Interest}

The authors declare that there are no conflicts of interest regarding the publication of this paper.

\section{Acknowledgments}

The authors would like to thank the Kanchanabhishek Institute of Medical and Public Health Technology, Praboromrajchanok Institute for Health Workforce Development, Ministry of Public Health, Thailand, for their funding and support of the current work. The authors are delighted to thank the National Institute of Child and Family for Development for supporting them with the EEG cap during the experimental period. They also gratefully thank Miss Kwanrutai Sampoon and Miss Sirirat Thongsri for helping with experimental setups. Most of all, they would like to thank the entire group of respondents who were the participants in this study and the parents for their understanding and cooperation.

\section{References}

[1] American Psychiatric Association, Diagnostic and Statistical Manual of Mental Disorders, DSM-5, American Psychiatric Association, Washington, DC, USA, 5th edition, 2013.
[2] E. Courchesne and K. Pierce, "Why the frontal cortex in autism might be talking only to itself: local over-connectivity but longdistance disconnection," Current Opinion in Neurobiology, vol. 15, no. 2, pp. 225-230, 2005.

[3] V. L. Cherkassky, R. K. Kana, T. A. Keller, and M. A. Just, "Functional connectivity in a baseline resting-state network in autism," NeuroReport, vol. 17, no. 16, pp. 1687-1690, 2006.

[4] G. M. McAlonan, V. Cheung, C. Cheung et al., "Mapping the brain in autism. A voxel-based MRI study of volumetric differences and intercorrelations in autism," Brain, vol. 128, no. 2, pp. 268-276, 2005.

[5] K. Nickel, L. Tebartz van, E. Elst et al., "Altered white matter integrity in adults with autism spectrum disorder and an IQ >100: a diffusion tensor imaging study," Acta Psychiatrica Scandinavica, vol. 135, no. 6, pp. 573-583, 2017.

[6] A. Mizuno, M. E. Villalobos, M. M. Davies, B. C. Dahl, and R. A. Müller, "Partially enhanced thalamocortical functional connectivity in autism," Brain Research, vol. 1104, no. 1, pp. 160174, 2006.

[7] P. Walsh, N. Kane, and S. Butler, "The clinical role of evoked potentials," Neurology in Practice, vol. 76, no. 2, pp. iil6-ii22, 2005.

[8] M. P. Philpot, D. Amin, and R. Levy, "Visual evoked potentials in Alzheimer's disease: correlations with age and severity," Electroencephalography and Clinical Neurophysiology/ Evoked Potentials, vol. 77, no. 5, pp. 323-329, 1990.

[9] G. Stothart, N. Kazanina, R. Naatanen, J. Haworth, and A. Tales, "Early visual evoked potentials and mismatch negativity in Alzheimer's disease and mild cognitive impairment," Journal of Alzheimer's Disease, vol. 44, no. 2, pp. 397-408, 2015.

[10] G. Coppola, M. Bracaglia, D. Di Lenola et al., "Visual evoked potentials in subgroups of migraine with aura patients," The Journal of Headache and Pain, vol. 16, no. 1, article 92, 2015.

[11] J. Jancic, I. Petrusic, V. Pavlovski, Z. Savkovic, D. Vucinic, and Z. Martinovic, "Pattern-reversal visual evoked potential parameters and migraine in the teenage population," Journal of Child Neurology, vol. 31, no. 6, pp. 717-721, 2015.

[12] A. Rady, A. Elsheshai, O. Elkholy, H. Abou El Wafa, and I. Ramadan, "P-1293 - Visual processing in schizophrenia assessed by visual evoked potential," European Psychiatry, vol. 27, p. 1, 2012.

[13] F. Khaliq, Y. Anjana, and N. Vaney, "Visual evoked potential study in slow learners," Indian Journal of Physiology and Pharmacology, vol. 53, no. 4, pp. 341-346, 2009.

[14] Y. Anjana, F. Khaliq, and N. Vaney, "Visual evoked potential in attention deficit/hyperactivity disorder," Delhi Psychiatry Journal, vol. 13, no. 2, pp. 294-297, 2010.

[15] A. Romani, S. Conte, R. Callieco et al., "Visual evoked potential abnormalities in dyslexic children," Functional Neurology, vol. 16, no. 3, pp. 219-229, 2001.

[16] I. S. Knoth, P. Vannasing, P. Major, J. L. Michaud, and S. Lippé, "Alterations of visual and auditory evoked potentials in fragile X syndrome," International Journal of Developmental Neuroscience, vol. 36, pp. 90-97, 2014.

[17] Y. Takarae, S. R. Sablich, S. P. White, and J. A. Sweeney, "Neurophysiological hyperresponsivity to sensory input in autism spectrum disorders," Journal of Neurodevelopmental Disorders, vol. 8, no. 1, article no. 29, 2016.

[18] American Psychiatric Association, Diagnostic and Statistical Manual of Mental Disorders, American Psychiatric Association, Washington, DC, USA, 4th edition, 2000. 
[19] R. C. Oldfield, "The assessment and analysis of handedness: the Edinburgh inventory," Neuropsychologia, vol. 9, no. 1, pp. 97-113, 1971.

[20] C. Pornnoppadol, A. Thongngern, A. Kewalin, and D. Sangrattanayon, "Development of the Pervasive Developmental Disorders Screening Questionnaire," Journal of the Psychiatric Association of Thailand, vol. 47, no. 2, pp. 75-96, 2002.

[21] B. Rimland and S. M. Edelson, The Autism Treatment Evaluation Checklist (ATEC), http://www.autism.com/ind_atec.

[22] W. Muangmolmaneerut, The relationship between selected factors, social support and dependent care agency among mothers of autistic children [Thesis of Master of Nursing Science (Mental Health and Psychiatric Nursing)], Faculty of Graduate Studies, Chiang Mai University, Chiang Mai, Thailand, 2004.

[23] S. S. Sparrow, D. V. Cicchetti, and D. A. Balla NCS Pearson, Inc., Minneapolis, Minnesota, USA, 2th edition, 2005.

[24] H. H. Jasper, "The ten-twenty electrode system of the International Federation," Electroencephalography and Clinical Neurophysiology, vol. 10, pp. 371-375.

[25] H. S. M. Kiiski, S. N. Riada, E. C. Lalor et al., "Delayed P100Like latencies in multiple sclerosis: A preliminary investigation using visual evoked spread spectrum analysis," PLOS ONE, vol. 11, no. 1, article no. A35, 2016.

[26] A. van der Walt, S. Kolbe, P. Mitchell et al., "Parallel changes in structural and functional measures of optic nerve myelination after optic neuritis," PLoS ONE, vol. 10, no. 5, Article ID e0121084, 2015.

[27] A. Zaher, "Visual and Brainstem Auditory Evoked Potentials in Neurology," in Methods for Evaluating Muscle and Nerve Function, M. Schwartz, Ed., InTech, 2012.

[28] J. M. P. Baas, J. L. Kenemans, and G. R. Mangun, "Selective attention to spatial frequency: An ERP and source localization analysis," Clinical Neurophysiology, vol. 113, no. 11, pp. 18401854, 2002.

[29] C. R. Bird, M. Hedberg, B. P. Drayer, P. J. Keller, R. A. Flom, and J. A. Hodak, "MR assessment of myelination in infants and children: usefulness of marker sites," American Journal of Neuroradiology, vol. 10, no. 4, pp. 731-740, 1989.

[30] T. Paus, D. L. Collins, A. C. Evans, G. Leonard, B. Pike, and A. Zijdenbos, "Maturation of white matter in the human brain: A review of magnetic resonance studies," Brain Research Bulletin, vol. 54, no. 3, pp. 255-266, 2001.

[31] J. Kornmeier, R. Wörner, A. Riedel, M. Bach, and L. Tebartz Van Elst, "A different view on the checkerboard? Alterations in early and late visually evoked EEG potentials in Asperger observers," PLoS ONE, vol. 9, no. 3, Article ID e90993, 2014.

[32] E. Neuhaus, A. Kresse, S. Faja, R. A. Bernier, and S. J. Webb, "Face processing among twins with and without autism: Social correlates and twin concordance," Social Cognitive and Affective Neuroscience, vol. 11, no. 1, Article ID nsv085, pp. 44-54, 2015.

[33] P. M. Siper, V. Zemon, J. Gordon et al., "Rapid and objective assessment of neural function in autism spectrum disorder using transient visual evoked potentials," PLoS ONE, vol. 11, no. 10, Article ID e0164422, 2016.

[34] L. A. Dion, G. Muckle, C. Bastien, S. W. Jacobson, J. L. Jacobson, and D. Saint-Amour, "Sex differences in visual evoked potentials in school-age children: What is the evidence beyond the checkerboard?" International Journal of Psychophysiology, vol. 88, no. 2, pp. 136-142, 2013.

[35] R. Sharma, S. Joshi, K. D. Singh, and A. Kumar, "Visual evoked potentials: Normative values and gender differences," Journal of Clinical and Diagnostic Research, vol. 9, no. 7, pp. 12-15, 2015. 


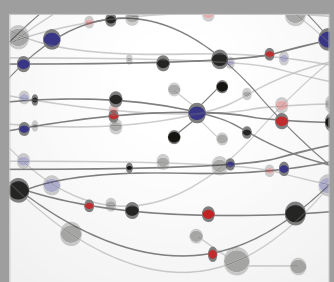

The Scientific World Journal
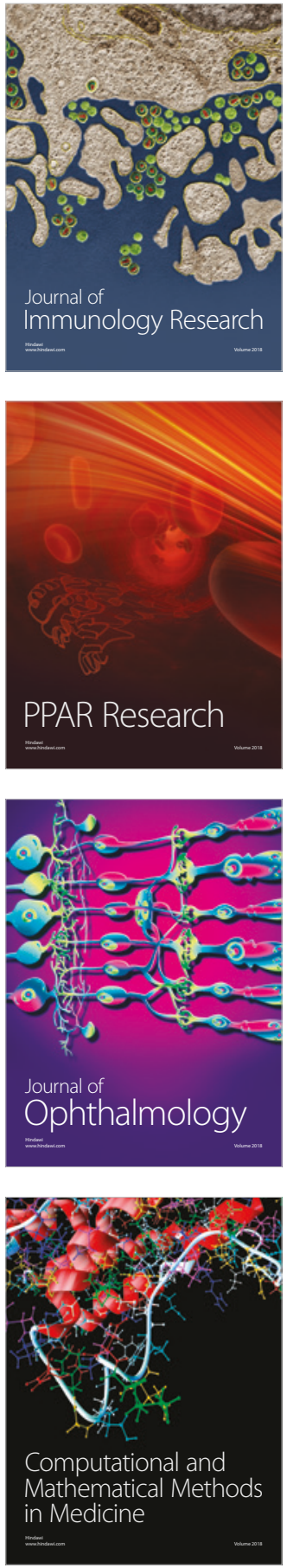

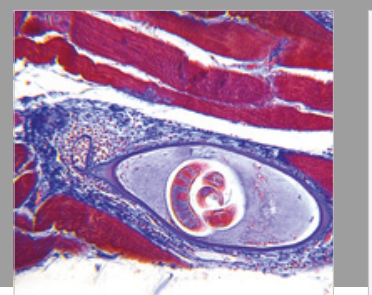

Gastroenterology Research and Practice

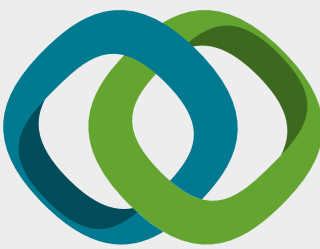

\section{Hindawi}

Submit your manuscripts at

www.hindawi.com
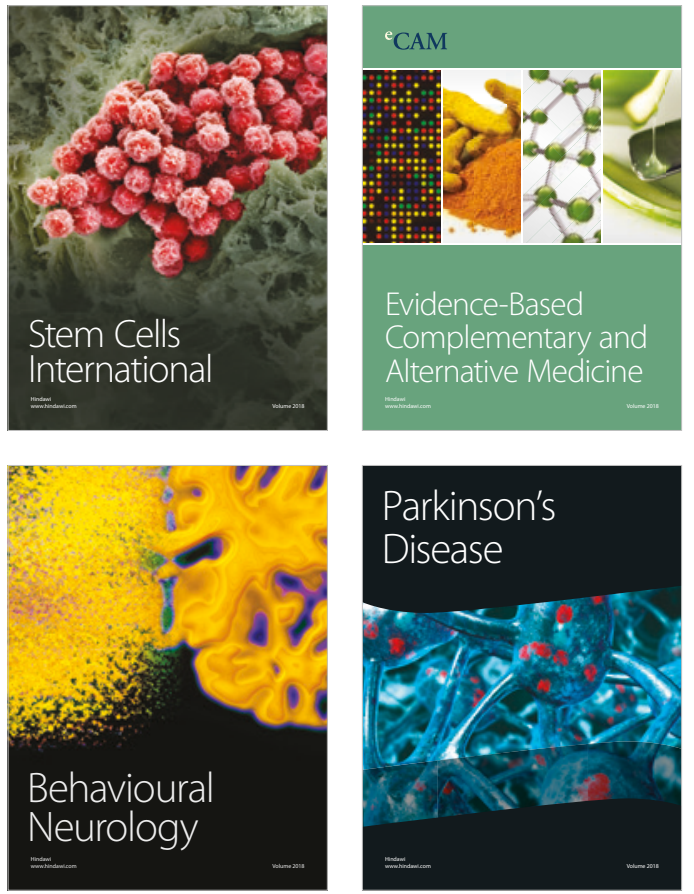

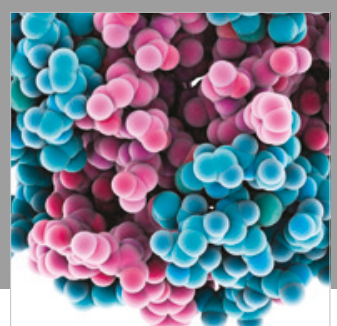

ournal of

Diabetes Research

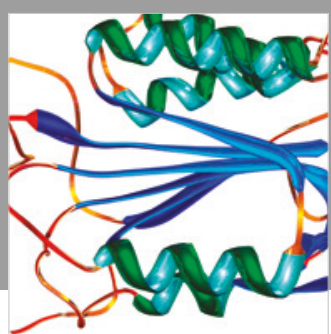

Disease Markers
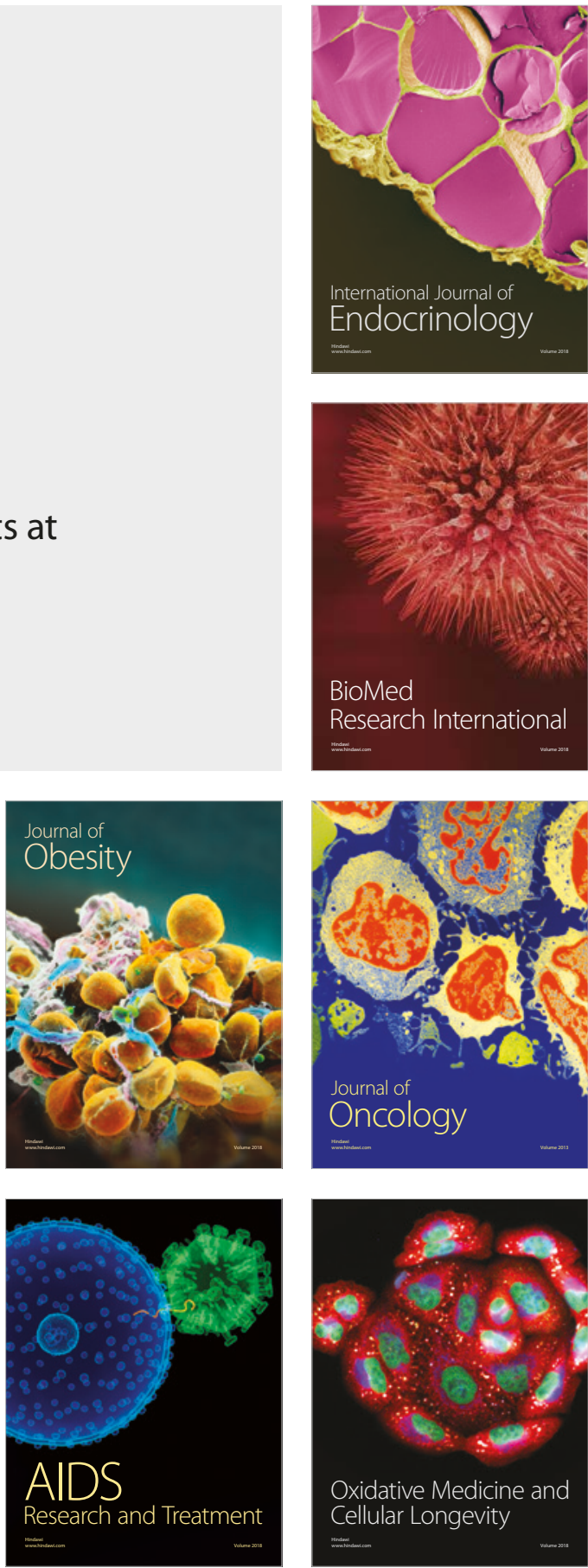